\title{
Cavernoma dural de fosa media Reporte de un caso y revisión de la literatura
}

\author{
Nicole Loyola ${ }^{1}$, Leonidas Quintana ${ }^{1,2}$, Paolo Massaro², Rodrigo Riveros ${ }^{3}$, Francisco Torres ${ }^{3}$, Humberto Vallejos ${ }^{4}$. \\ Catedra de Neurocirugía. Escuela de Medicina. Universidad de Valparaíso. \\ Servicio de Neurocirugía. Hospital Carlos Van Buren. \\ 3 Servicio de Neurorradiología. Hospital Carlos Van Buren. \\ 4 Servicio de Anatomía Patológica. Hospital Carlos Van Buren.
}

Rev. Chil. Neurocirugía 44: 150-153, 2018

\section{Resumen}

Introducción: Los cavernomas durales son lesiones raras. El principal diagnóstico diferencial son los meningiomas. Caso clínico: Mujer de 20 años que presenta cuadro de crisis convulsivas generalizadas. La resonancia muestra una lesión extraaxial del ala esfenoidal izquierda con características de meningioma. Se sometió a cirugía encontrando una masa blanda, rojiza y vascularizada. La biopsia informa cavernoma dural. Discusión: Los cavernomas durales son histológicamente idénticos a los parenquimatosos, pero difieren en su presentación clínica y características imagenológicas. Deben incluirse en el diagnóstico diferencial de lesiones extra-axiales de base dural. Se comportan como lesiones tumorales vascularizadas por lo cual su principal tratamiento es la cirugía.

Palabras clave: Cavernoma, meningeoma.

\begin{abstract}
Introduction: Dural cavernomas are non-usual lesions. The main differential diagnosis is meningiomas. Clinical case: A 20-year-old woman presented with generalized seizures. The resonance shows an extraaxial lesion in the left sphenoid wing with characteristics of meningioma. At surgery was found a soft, reddish and vascularized mass. The biopsy reports dural cavernoma. Discussion: Dural cavernomas are histologically identical to parenchymatous ones, but they differ in their clinical presentation and imaging characteristics. They should be included in the differential diagnosis of dural-based extra-axial lesions. They behave as vascularized tumor lesions, which is why their main treatment is surgery.
\end{abstract}

Key words: Cavernous hemangioma, meningioma.

\section{Introducción}

Los cavernomas, también llamados angiomas o hemangiomas cavernosos, constituyen el 5 a $13 \%$ de las malformaciones vasculares cerebrales ${ }^{1,2}$. Son hamartomas vasculares benignos, con espacios sinusoidales de paredes delgadas revestidos con endotelio. Pueden encontrarse en cualquier área del sistema nervioso central, pero son más frecuentes en la sustancia blanca subcortical hemisférica ${ }^{3}$. Menos frecuentemente tienen localización extracerebral, donde son conocidos como cavernomas durales, constituyendo un $0,4-2 \%$ de estas malformaciones ${ }^{8}$. Se describen 2 localizaciones: los de fosa media, y los ubicados fuera esta (la hoz, tentorio, convexidad, fosa anterior, ángulo pontocerebeloso o cavum) los cuales son aún más raros ${ }^{1,3,8}$. Las características clínicas de ambos grupos difieren, teniendo un compor- tamiento más agresivo los de fosa media, secundario a su vascularización ${ }^{3}$. Los primeros reportes mostraban gran morbi-mortalidad secundaria a sangrado intra operatorio9, correspondiendo estas descripciones exclusivamente a cavernomas del seno cavernoso. En el presente caso clínico se revisan aspectos clínicos, histológicos e imagenológicos de los cavernomas durales, sus diferencias con los cavernomas parenquimatosos y los hallazgos que llevan a 
diagnosticarlos como meningiomas en el estudio pre operatorio.

\section{Caso clínico}

Se presenta el caso de una mujer de 20 años, sin antecedentes mórbidos. Consulta por cuadro de crisis convulsiva generalizada seguida de cefalea y vómitos 2 días previo al ingreso. Sin déficit neurológico focal.

La resonancia magnética $(1,5 \mathrm{~T})$ mostró una lesión expansiva extra-axial en relación al ala esfenoidal izquierda, con señal hipointensa en T1-weighted, hiperintensa en T2-weighted, con captación de contraste intenso y heterogéneo (Figura 1). Presenta efecto de masa, con desplazamiento de la arteria cerebral media y sus ramas.

Se realizó una angiografía cerebral de 4 vasos que mostró una masa avascular de la fosa media izquierda, con desplazamiento la arteria cerebral media, sin aferencias durales. (Figura 2).

Con el diagnóstico presuntivo de meningioma del ala esfenoidal se realizó la cirugía de exéresis tumoral, utilizando un abordaje pterional izquierdo. Tras la apertura dural se observó una lesión rojo-vinosa brillante con un claro plano de clivaje con el parénquima cerebral. Se procedió a la desvascularización precoz con bipolar, sin apertura de la capsula, seguida de la exéresis en bloque. La pieza quirúrgica era de consistencia firme, con una superficie externa abollonada. En la superficie de corte, se observa una masa esponjosa llena material hemático (Figura 3).

El estudio histológico con tinción de hematoxilina eosina (HE) mostró aglomeración de vasos sanguíneos de distinto tamaño, separados por paredes delgadas de tejido fibroso. Algunos vasos presentan fibrina en su interior (trombosis). (Figura 4). Los hallazgos descritos son concordantes con un cavernoma dural de fosa media.

\section{Discusión}

Los cavernomas durales, histológicamente, son idénticos a los parenquimatosos, pero difieren en su comportamiento y características imagenológicas $^{8}$. Los cavernomas parenquimatosos crecen en relación a hemorragias recurrentes, produciendo cefalea, convulsiones o un déficit neurológico

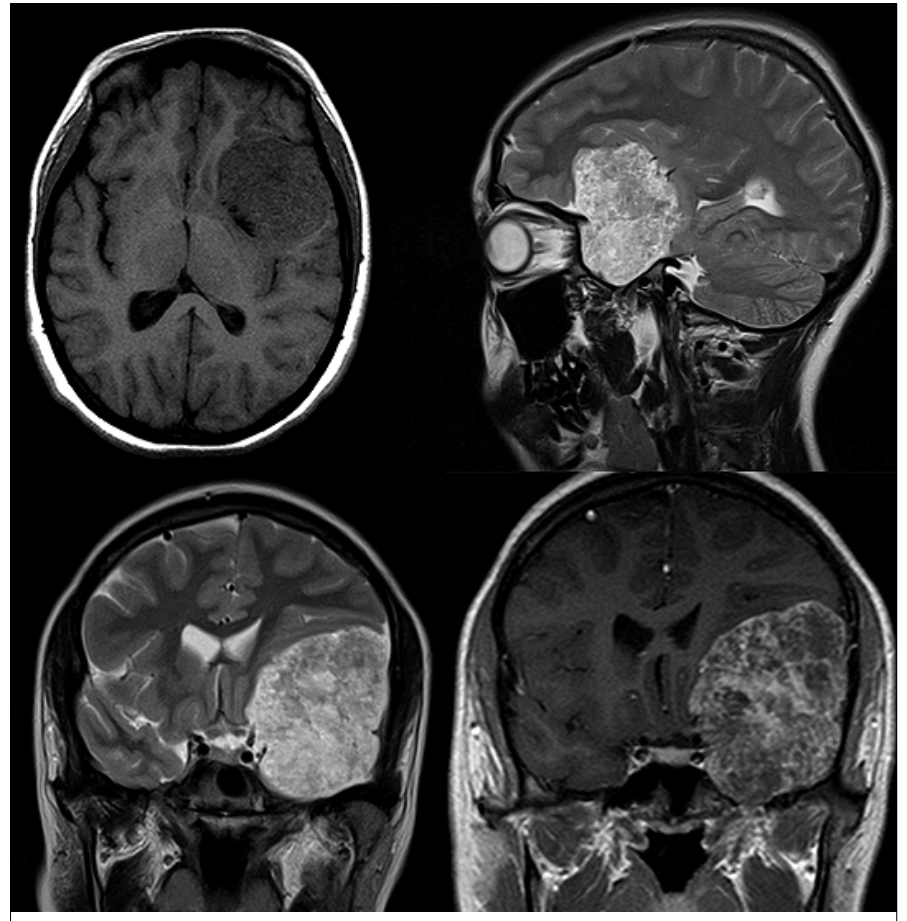

Figura 1. Resonancia Magnética. Imagen superior izquierda: corte axial, señal Hipointensa en T1-weighted. Imagen superior derecha: corte sagital, marcada señal hiperintensa en T2- weighted. Imagen inferior izquierda: corte coronal, señal hiperintensa heterogénea en T2- weighted. Imagen inferior derecha: corte coronal, intensa y heterogénea captación de contraste T1-Gd, sin cola dural.

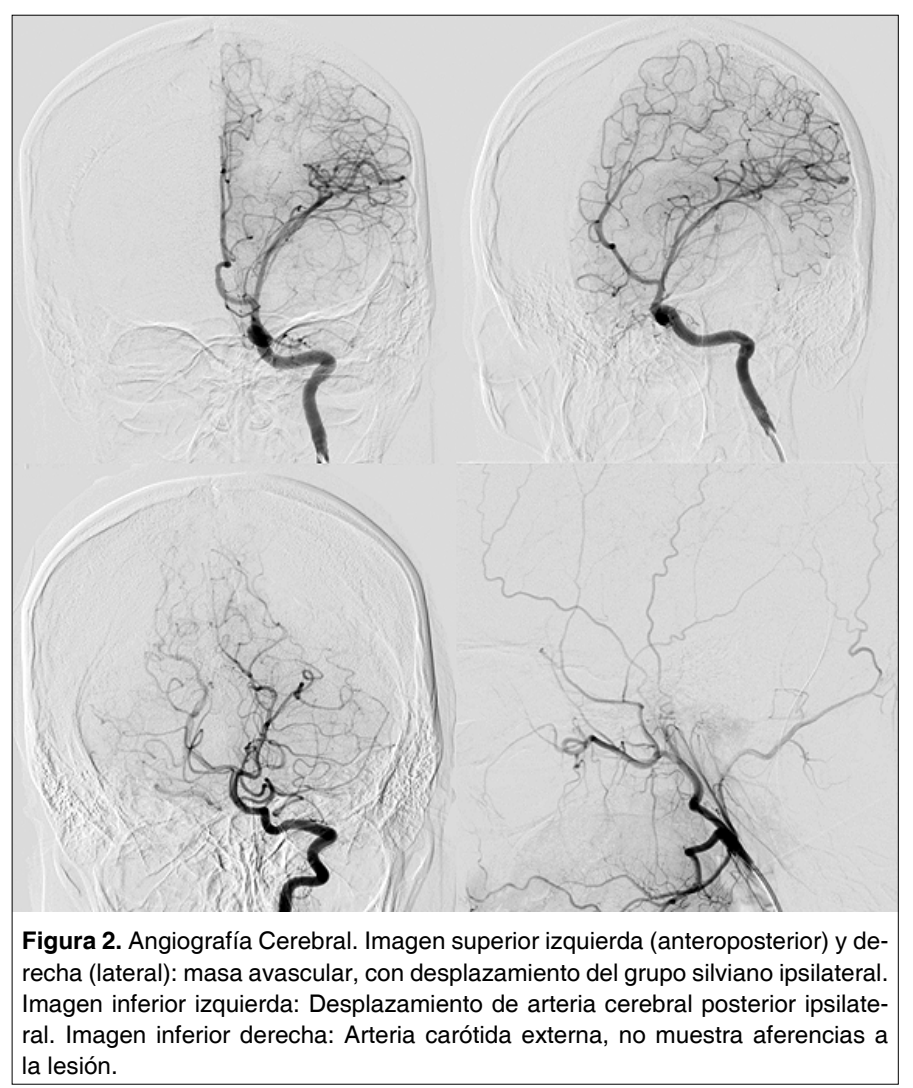




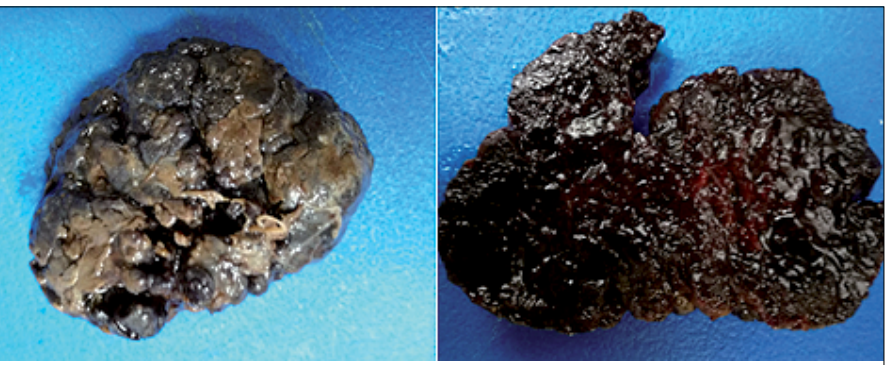

Figura 3. Pieza quirúrgica. Imagen izquierda: superficie externa, abollonada. Imagen derecha: superficie de corte, masa esponjosa.

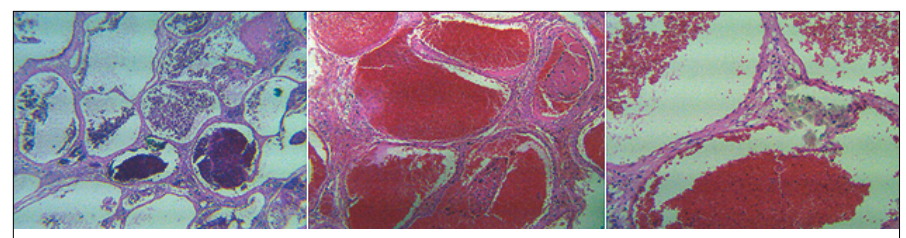

Figura 4. Microscopia con tinción hematoxilina eosina. Aumento 2,5x ; 4x y 10x respectivamente.

\begin{tabular}{l|l|l|}
$\begin{array}{l}\text { Tabla 1. } \\
\text { Características imegenológicas }\end{array}$ & $\begin{array}{l}\text { Cavernoma } \\
\text { parenquimatoso }\end{array}$ \\
\hline Tomografía & $\begin{array}{l}\text { Cavernoma dural } \\
\text { Iso- hiperdenso } \\
\text { Realce con contraste intenso yo yóneo } \\
\text { homogéneo }\end{array}$ & $\begin{array}{l}\text { Mínimo o nulo realce con } \\
\text { contraste }\end{array}$ \\
\hline Resonancia & $\begin{array}{l}\text { Iso - hipointenso en T1 } \\
\text { Hiperintenso en T2 } \\
\text { Realce intenso T1+Gd } \\
\text { Sin hemosiderina }\end{array}$ & $\begin{array}{l}\text { Señal heterogénea T1 } \\
\text { y T2 } \\
\text { Tipo "popcorn" } \\
\text { Sin realce de contraste } \\
\text { Anillo hemosiderina }\end{array}$ \\
\hline Angiografía & $1 / 3$ oculta. 2/3 blush & Oculta \\
\hline
\end{tabular}

progresivo $^{3}$. A su vez los cavernomas durales rara vez se presentan como hemorragia $^{3}$. Se cree que estos crecen por otros mecanismos como factores hormonales, ectasia o trombosis de los espacios perivasculares ${ }^{3,15}$. Se han descrito casos de crecimiento en relación al embarazo o inicio de terapia hormonal ${ }^{12,13}$. Los cavernomas durales son más frecuentes en mujeres, entre la 4-6을 década y en población japone$\mathrm{sa}^{4}$. En la Tabla 1 se resumen las principales diferencias imagenológicas entre los cavernomas parenquimatosos y durales.

Lewis et al. ${ }^{3}$ en 1994 clasificó los cavernomas durales en 2 grupos: de fosa media y de otras localizaciones. Los cavernomas durales de fosa media son más frecuentes y tienen un comportamiento más agresivo debido a su suministro vascular, pudiendo provocar déficits de campo visual, oculomotilidad, alteraciones hormonales y daño del trigémino ${ }^{3,4}$. Menos frecuente que lo visto en los parenquimatosos, pueden presentar crisis convulsivas ${ }^{1,13}$, como en el caso expuesto. Los cavernomas durales de otras ubicaciones se presentan más frecuentemente con cefalea $^{3}$. Existe solo un caso reportado de hemorragia aguda, con un hematoma subdural secundario a un cavernoma dural ${ }^{11}$.

En la tomografía computada, los cavernomas durales se presentan como masas isodensas a moderadamente hiperdensas con captación de contraste intensa y homogénea por lo son indistinguibles de un meningioma por esta técnica ${ }^{1,3,8}$. Pueden presentar calcificaciones $^{14}$.

Los cavernomas de fosa media pueden mostrar vascularización intensa en la angiografía, lo que es menos frecuente en otras localizaciones. En general se estima que $1 / 3$ de las lesiones son ocultas en la angiografía cerebral, mientras que $2 / 3$ muestran cierto grado de "rubor" (blush) dado por los vasos de alimentación tumoral desde la arteria carótida cavernosa o carótida externa ${ }^{3,4,12}$. Por el contrario, los meningiomas típicamente presentan un pedículo vascular con vasos radiales ("sunburst") en angiografía ${ }^{5,8}$. En el caso reportado, a pesar del gran volumen de la lesión, se presentó como una masa avascular.

En resonancia magnética, los cavernomas durales muestran señal iso 0 hipointensa en T1-weighted ${ }^{10,8}$, en T2weighted son frecuentemente hiperintensos ${ }^{3,4}$, aunque también pueden presentar señal mixta ${ }^{10}$. Tienen realce intenso y homogéneo tras la administración de gadolinio, pudiendo presentar cola dural ${ }^{3,10,14}$. El halo periférico hipointenso en T2-weighted por depósito de hemosiderina, característico de los cavernomas parenquimatosos, no está presente en los durales ${ }^{8}$. Generalmente, los meningiomas son iso o ligeramente hiperintensos en T2-weighted, por lo cual la hiperintensidad marcada en T2-weighted constituye la principal herramienta para el diagnóstico diferencial entre ambas entidades ${ }^{4,8,13,15}$.

Los cavernomas durales, a diferencia de los parenquimatosos, se comportan más como un tumor con efecto de masa y crecimiento documentado en series de $\operatorname{casos}^{12,15}$. Lo anterior sumado a una historia natural desconocida, hacen que la resección quirúrgica constituya el tratamiento de elección en todas las lesiones sintomáticas ${ }^{8,12,15}$. Los cavernomas de fosa media presentan un desafío técnico debido a su adherencia a estructuras neurovasculares y su irrigación desde la arteria carótida cavernosa o ramas de la arteria carótida externa, todo lo cual puede llevar a una resección parcial por sangrado no controlado ${ }^{3,13}$. Algunos autores proponen embolización ${ }^{6}$ o radioterapia previa para disminuir el sangrado operatorio. Shibata et al., fue el primero en descri- 
bir la radiosensibilidad de estas lesiones, publicando la reducción del tamaño lesional con radioterapia externa, lo cual no se observa en cavernomas parenquimatosos ${ }^{7,8}$. A su vez los cavernomas de otras localizaciones en general no requieren de tratamiento pre operatorio ${ }^{3,13}$. En el caso expuesto, la devascularización precoz sin apertura de la capsula, permitió una exéresis en bloque con mínima perdida sanguínea, lo que se condice con lo publicado por otros autores ${ }^{8,15}$.

\section{Conclusión}

Los cavernomas durales son lesiones raras, pero que deben incluirse en el diagnóstico diferencial de lesiones ex- tra-axiales. La hiperintesidad marcada en T2-weighted constituye una característica a considerar. La sospecha diagnóstica permite adecuar la estrategia quirúrgica y con ella disminuir la probabilidad de sangrado no controlable durante la resección.

Recibido: 18 de abril de 2018

Aceptado: 31 de mayo de 2018

\section{Referencias}

1. Isla A. Intracranial Cavernous Angioma in the Dura. Neurosurgery. 1989 (4): 657-659.

2. Del CO Jr. An analysis of the natural history of cavernous angiomas. J Neurosurg 1991. 75 (5): 702-708.

3. Lewis, Al. Dural cavernous angiomas outside the middle cranial fossa: a report of two cases. Neurosurgery. 1994 Sep;35(3): 498-504.

4. Momoshima S. MR findings in extracerebral cavernous angioma of the middle cranial fossa: Report of two cases and review of the literature. AJNR 12: 756-760, 1991.

5. Steven W. Hwang. Convexity dural cavernous malformation with intradural and extradural extension mimicking a meningioma: a case report. Acta Neurochir (2009) 151: 79-83.

6. Namba S. Extracerebral cavernous hemangioma of the middle cranial fossa. Surg Neurol 19: 379-388, 1983.

7. Shibata S, Mori K. Effect of radiation therapy on extracerebral cavernous hemangioma in the middle fossa. J Neurosurg 67: 919-922, 1987.

8. Melone, A. Intracranial Extra-axial Cavernous Angioma of the Cerebellar Falx. World Neurosurgery 74 [4/5]: 501-504, 2010.

9. Linskey ME, Sekhar LN: Cavernous sinus hemangiomas: a series, a review, and an hypothesis. Neurosurgery 30:101-108, 1992

10. Tsutsumi S, Yasumoto Y, Saeki H, et al. Cranial Dural Cavernous Angioma. Clin Neuroradiol (2014) 24: 155.

11. Suzuki K, Kamezaki T, Tsuboi K, Kobayashi E. Dural cavernous angioma causing acute subdural hemorrhage-case report. Neurol Med Chir (Tokyo). 1996; 36: 580-2.

12. González LF. Are cavernous sinus hemangiomas and cavernous malformations different entities? Neurosurg Focus. 2006 Jul 15; 21(1).

13. Rosso D. Dural Cavernous Angioma: A Preoperative Diagnostic Challenge. Can. J. Neurol. Sci. 2003; 30: $272-277$.

14. Vogler R. Dural Cavernous Angioma: MR Features. AJNR 16: 773-775, Apr 1995.

15. Meyer F. Extra-axial cavernous hemangiomas involving the dural sinuses. J Neurosurg 73: 187-192, 1990.

\section{Correspondencia a:}

Dra. Nicole Loyola

Neurocirujana, Hospital de Coyhaique, Chile.

Nicole.loyola@gmail.com 